\title{
Is there any correlation between liver graft regeneration and recipient's pretransplant skeletal muscle mass? - a study in extended left lobe graft living-donor liver transplantation
}

\author{
Riccardo Pravisani ${ }^{1,2}$, Akihiko Soyama ${ }^{1}$, Shinichiro Ono ${ }^{1}$, Umberto Baccarani ${ }^{2}$, Miriam Isola ${ }^{3}$, \\ Mitsuhisa Takatsuki ${ }^{1}$, Masaaki Hidaka ${ }^{1}$, Tomohiko Adachi ${ }^{1}$, Takanobu Hara ${ }^{1}$, Takashi Hamada ${ }^{1}$, \\ Florian Pecquenard ${ }^{1}$, Andrea Risaliti ${ }^{2}$, Susumu Eguchi ${ }^{1}$
}

${ }^{1}$ Department of Surgery, Nagasaki University Graduate School of Biomedical Sciences, Nagasaki, Japan; ${ }^{2}$ Liver-Kidney Transplant Unit, Department of Medicine, ${ }^{3}$ Division of Medical Statistic, Department of Medicine, University of Udine, Udine, Italy

Contributions: (I) Conception and design: R Pravisani, S Ono, M Hidaka, S Eguchi; (II) Administrative support: S Eguchi; (III) Provision of study material or patients: A Soyama, S Ono, M Takatsuki, M Hidaka, T Adachi, T Hara, T Hamada, S Eguchi; (IV) Collection and assembly of data: R Pravisani, A Soyama, T Adachi, T Hara, T Hamada, F Pecquenard; (V) Data analysis and interpretation: R Pravisani, S Eguchi, U Baccarani, S Ono, M Hidaka, M Isola; (VI) Manuscript writing: All authors; (VII) Final approval of manuscript: All authors.

Correspondence to: Susumu Eguchi, MD, PhD, FACS, FEBS. Department of Surgery, Nagasaki University Graduate School of Biomedical Sciences, 1-7-1 Sakamoto, Nagasaki 852-8501, Japan. Email: sueguchi@nagasaki-u.ac.jp.

Background: The end-stage liver disease causes a metabolic dysfunction whose most prominent clinical feature is the loss of skeletal muscle mass (SMM). In living-donor liver transplantation (LDLT), liver graft regeneration (GR) represents a crucial process to normalize the portal hypertension and to meet the metabolic demand of the recipient. Limited data are available on the correlation between pre-LDLT low SMM and GR.

Methods: Retrospective study on a cohort of 106 LDLT patients receiving an extended left liver lobe graft. The skeletal muscle index (SMI) at L3 level was used for muscle mass measurement, and the recommended cut-off values of the Japanese Society of Hepatology guidelines were used as criteria for defining low muscularity. GR was evaluated as rate of volume increase at 1 month post-LT [graft regeneration rate (GRR)]. Results: The median GRR at 1 month post-LT was 91\% (IQR, 65-128\%) and a significant correlation with graft volume-to-recipient standard liver volume ratio (GV/SLV) (rho -0.467, $\mathrm{P}<0.001$ ), graft-torecipient weight ratio (GRWR) (rho $-0.414, \mathrm{P}<0.001$ ), donor age (rho $-0.306, \mathrm{P}=0.001$ ), 1 month post-LT cholinesterase serum levels (rho 0.397, $\mathrm{P}=0.002$ ) and pre-LT low muscularity [absent vs. present GRR 97.5\% (73.1-130\%) vs. 83.5\% (45.2-110.9\%), $\mathrm{P}=0.041$ ] was noted. Moreover in male recipients, but not in women, it was shown a direct correlation with pre-LT SMI (rho 0.352, P=0.020) and inverse correlation with 1 month post-LT SMI variation (rho -0.301, $\mathrm{P}=0.049$ ). A low $\mathrm{GRR}$ was identified as an independent prognostic factor for recipient overall survival (HR 6.045, $\mathrm{P}<0.001$ ).

Conclusions: Additionally to the hemodynamic factors of portal circulation and the quality of the graft, the metabolic status of the recipients has a significant role in the GR process. A pre-LT low SMM is associated with impaired GRR and this negative impact is more evident in male recipients.

Keywords: Liver regeneration; living-donor liver transplantation (LDLT); skeletal muscle mass index; sarcopenia; malnutrition

Submitted Mar 22, 2019. Accepted for publication May 16, 2019.

doi: $10.21037 / \mathrm{hbsn} .2019 .11 .08$

View this article at: http://dx.doi.org/10.21037/hbsn.2019.11.08 


\section{Introduction}

Sarcopenia, defined as a pathological reduction of skeletal muscle mass and strength, is one of the most clinically impactful features of the metabolic dysfunction related to end stage liver disease (ESLD) (1). It is associated with an increased morbidity and mortality, poor performance status and quality of life both before and after liver transplantation (LT) (1-3). Unfortunately, it has been verified that LT does not invariably guarantee a curative effect on sarcopenia with no recovery of the skeletal muscle mass $(4,5)$. Moreover, the surgical stress and complications related to LT may even further compromise and worsen such metabolic dysfunction, particularly in the early postoperative period.

In living-donor liver transplantation (LDLT), liver graft regeneration represents a crucial process to normalize the portal hypertension associated with small-for-size syndrome and to meet the metabolic and biosynthetic demand of the recipient (6). In clinical setting, graft regeneration has been mainly correlated with hemodynamic factors and the quality of the graft (7-11). However, graft regeneration is surely an anabolic process which may also depend on the adequate availability of energy and metabolites.

Therefore, the aim of the present study was to investigate whether the recipient's skeletal muscle mass measured at the pre-LT and early post-LT phase showed any association with the graft regeneration at 1 month post-LT, in left lobe graft LDLT.

\section{Methods}

\section{Skeletal muscle mass measurement}

To evaluate the presence and severity of low muscularity, the skeletal muscle index (SMI) at the lower end plate of the L3 body (L3-SMI) was used, as previously reported (12). CT images were analyzed with SYNAPSE VINCENT (FUJIFILM, Tokyo, Japan). The L3 SMI was expressed as cross-sectional muscle area/height ${ }^{2}$, and the cut-off for a diagnosis of low muscle mass was L3-SMI $<42 \mathrm{~cm}^{2} / \mathrm{m}^{2}$ for men and L3-SMI $<38 \mathrm{~cm}^{2} / \mathrm{m}^{2}$ for women, as determined by the Japanese Society of Hepatology guidelines (13). The SMI variation $(\mathrm{SMIv} \%)$ was calculated using the following formula:

$$
\text { SMIv \% }=\left(\mathrm{SMI}_{1 \text { month post-LT }}-\mathrm{SMI}_{\text {pre-LT }}\right) \times 100 / \mathrm{SMI}_{\text {pre-LT }}
$$

Body mass index (BMI) as anthropometric parameter was calculated the day before LT procedure. No weight correction was done in presence of ascites.

\section{Liver regeneration}

For 3-dimentional volumetry reconstruction, $0.5-3 \mathrm{~mm}$ thick images acquired during a 3 phase (arterial, portal, venous) dynamic multidetector computer tomography (CT) scan were analyzed with SYNAPSE VINCENT (FUJIFILM, Tokyo, Japan). In donors, after the reconstruction of the whole liver volume and vessels, the virtual liver partition was performed and the graft volume (GV) measured. In recipients, the whole GV was measured at 1 month post-LT with the same methodology. The graft regeneration rate (GRR) was calculated using the following formula (14):

$$
\text { GRR\% }=\left(G V_{\text {lmonth post-LT }}-G V_{\text {pre-LT }}\right) \times 100 / G V_{\text {pre-LT }}
$$

Both SMI and GV were measured by a single researcher, specifically trained in SYNAPSE VINCENT use, under the direct supervision of the surgeons who actually managed the patients clinically. Only Pre-LT CT scans performed within 3 months before LDLT were used in the analysis.

\section{Patients characteristics}

From January 2008 to October 2018, a total of 190 patients underwent primary LDLT at the Nagasaki University Hospital. Technical details about the surgical procedure applied for LDLT have already been described elsewhere (15). Right lobe grafts or right posterior sector grafts $(n=66)$ were excluded from the analysis to remove the confounding effect of hepatic venous congestion which has a known negative impact on regeneration and is variably related to the middle hepatic vein management in the graft (middle hepatic vein inclusion or not, middle hepatic vein tributaries reconstruction or not). Pediatric cases were excluded $(n=4)$. Recipients with an overall survival lower than 1 month, graft loss within the first post-LT month or unavailability of CT scan at 1 month post LT were excluded from the analysis as they could not be evaluated in terms of graft regeneration $(\mathrm{n}=14)$ (Figure 1). Aside from the skeletal muscle mass, early major postoperative complications were also investigated as potential determinants of graft regeneration. They were considered when occurring within the first post-LT month and were defined as follows:

- Re-laparotomy: any surgical complication requiring relaparotomy;

- Vascular complication: any arterial or venous (portal and hepatic veins) complication;

- Biliary complication: biliary leakage, stricture or 


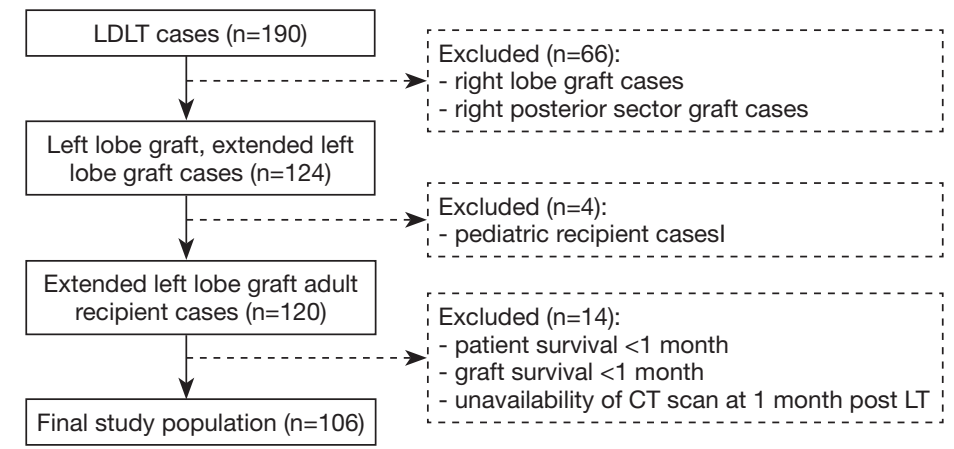

Figure 1 Flowchart describing the study population selection.

cholangitis;

- Blood stream infection: clinical and laboratory features of sepsis associated with positive hemocultures;

- Graft rejection: any case (antibody mediated rejection, cell mediated rejection) of histopathologically verified graft rejection.

All patients were managed with an early enteral feeding (EEF) protocol using a tube jejunostomy placed at the time of LT, as previously reported (12). Briefly, A tube jejunostomy was placed at the time of LT, and 24 hours postoperatively, enteral nutrition with Elental (Ajinomoto Pharmaceutical Ltd, Tokyo, Japan) was introduced. The calories load was $1 \mathrm{kcal} / \mathrm{mL}$ and the initial infusion rate was $10 \mathrm{~mL} / \mathrm{h}$. If the patient tolerated the enteral load well, the rate was increased up to $60 \mathrm{~mL} / \mathrm{h}$ and maintained until sufficient oral intake was possible. Extubation was usually performed on postoperative day (POD) 1-2 and oral intake was usually initiated at POD 4-5. Enteral nutrition was reduced progressively and discontinued when the oral intake exceeded 1,200 kcal/day. None of the patients required unplanned complete withdrawal of enteral feeding due to side effects, nor did any jejunostomy-related complications occur. The standard immunosuppression regimen comprised tacrolimus and steroids. The steroids were gradually tapered and discontinued by 3 months after LDLT. Mycophenolate mofetil was added for ABOincompatible LDLT patients and patients who were intentionally kept at lower trough levels of tacrolimus due to renal dysfunction. A detailed protocol has been described elsewhere (15).

\section{Statistical analysis}

Categorical variables were expressed as frequencies and percentage, while continuous variables were expressed as mean \pm standard deviation $(\mathrm{SD})$ or median [interquartile range (IQR)], as appropriate. For categorical variables, cross-tabulations were generated, and the chi-square or Fisher's exact test was used to compare distributions.

Differences in terms of graft regeneration according to categorical variables were analysed by Student's $t$ or Mann-Whitney test, as appropriate. Pearson or Spearman correlation coefficients were used to explore any correlation between graft regeneration and continuous variables. Multivariate linear regression analysis was performed including all variables significant at $\mathrm{P}<0.05$ in the aforementioned analysis.

Graft loss was defined as death due to graft failure or retransplantation. Death with functioning graft was considered as a competing risk event because death for causes unrelated to graft loss precludes the occurrence of graft loss. The cumulative incidence method was used to estimate graft loss accounting for the presence of competing risks. Based on the method of Fine and Gray, univariate and multivariate competing-risk regression were used to explore which factors were associated with graft loss. This model is based on the hazard of the subdistribution and provides a simple relationship between covariates and cumulative incidence. The risk of multicollinearity was evaluated by means of the variance inflation factor. Variables of $\mathrm{P}$ less than 0.05 during univariate analysis were included in multivariable analysis.

The OS was defined as the time (months) from LT to either death or last observation and was described using the Kaplan-Meier approach. Univariate and multivariate Cox regression were used to estimate prognostic variables associated with OS, after the assumption of the proportional hazard was verified. The proportional hazard assumption was tested using the Schoenfeld residual test.

To investigate the impact of GRR on recipient OS and 
Table 1 Demographic, clinical characteristics of recipients and donors, intraoperative details and postoperative clinical course

\begin{tabular}{|c|c|}
\hline Characteristics & Total $(n=106)$ \\
\hline \multicolumn{2}{|l|}{ Recipient characteristics } \\
\hline Age (years) & 57 [50-62] \\
\hline $\operatorname{Sex}(M: F)$ & 43:63 \\
\hline BMI & $22.9(20.8-26.5)$ \\
\hline \multicolumn{2}{|l|}{ Pre-LT SMI $\left(\mathrm{cm}^{2} / \mathrm{m}^{2}\right)$} \\
\hline Men & $47.2 \pm 9.1$ \\
\hline Women & $39.1 \pm 7.1$ \\
\hline MELD & 17 [13-23] \\
\hline \multicolumn{2}{|l|}{ Child-Pugh class (\%) } \\
\hline$A$ & $3(2.8 \%)$ \\
\hline $\mathrm{B}$ & $30(28.3 \%)$ \\
\hline $\mathrm{C}$ & $73(68.9 \%)$ \\
\hline HCV infection (\%) & $36(34.0 \%)$ \\
\hline HCC diagnosis (\%) & $36(34.0 \%)$ \\
\hline Pre-transplant diabetes (\%) & $27(25.5 \%)$ \\
\hline Pre-transplant ICU admission (\%) & $10(9.4 \%)$ \\
\hline SLV (mL) & $1,141.3 \pm 147.2$ \\
\hline ABO incompatibility (\%) & $22(20.8 \%)$ \\
\hline \multicolumn{2}{|l|}{ Donor and graft characteristics } \\
\hline Age (years) & 34 [27-42] \\
\hline GV (mL) & $471[411-531]$ \\
\hline GW (gr) & $407.5[350-466]$ \\
\hline GV/SLV\% & $40 \%(36-47 \%)$ \\
\hline GRWR & $0.78[0.66-0.92]$ \\
\hline \multicolumn{2}{|c|}{ Operative details, post-LT clinical course and outcome } \\
\hline Operative time (min) & 761 [705-859] \\
\hline Blood loss (g) & $6,450[3,300-11,400]$ \\
\hline Post-LT ICU length of stay (days) & $7[5-11]$ \\
\hline Early re-laparotomy (\%) & $17(16.0 \%)$ \\
\hline Early biliary complications (\%) & $12(11.3 \%)$ \\
\hline Early vascular complications (\%) & $11(10.4 \%)$ \\
\hline Early blood stream infection (\%) & $28(26.4 \%)$ \\
\hline Early rejection (\%) & $14(13.21 \%)$ \\
\hline
\end{tabular}

Table 1 (continued)
Table 1 (continued)

\begin{tabular}{|c|c|}
\hline Characteristics & Total $(n=106)$ \\
\hline \multicolumn{2}{|l|}{1 month post-LT SMI $\left(\mathrm{cm}^{2} / \mathrm{m}^{2}\right)$} \\
\hline Men & $43.8 \pm 8.9$ \\
\hline Women & $39.7 \pm 7.8$ \\
\hline \multicolumn{2}{|l|}{ SMlv (\%) } \\
\hline Men & $\begin{array}{c}-8.00 \% \\
(-12.28 \% \text { to }-3.67 \%)\end{array}$ \\
\hline Women & $\begin{array}{c}+2.55 \% \\
(-5.30 \% \text { to }+11.27 \%)\end{array}$ \\
\hline 1 month post-LT GV (mL) & $867[748-1,023]$ \\
\hline 1 month post-LT GRR (\%) & $\begin{array}{c}90.90 \% \\
(65.65-127.76 \%)\end{array}$ \\
\hline $\begin{array}{l}1 \text { month post-LT bilirubin serum level } \\
(\mathrm{mg} / \mathrm{dL})\end{array}$ & $1.6(0.9-4.95)$ \\
\hline 1 month post-LT PT-INR & $1.2(1.1-1.4)$ \\
\hline $\begin{array}{l}1 \text { month post-LT albumin plasma level } \\
(\mathrm{g} / \mathrm{dL})\end{array}$ & $3.0 \pm 0.6$ \\
\hline $\begin{array}{l}1 \text { month post-LT cholinesterase serum } \\
\text { level ( } \mathrm{U} / \mathrm{dL})\end{array}$ & $107.5 \pm 48.3$ \\
\hline
\end{tabular}

BMI, body mass index; GRR, graft regeneration rate; GRWR, graft to recipient weight ratio; GV/SLV\%, graft volume to recipient standard liver volume ratio; GW, graft weight; HCV, hepatitis $\mathrm{C}$ virus; HCC, hepatocellular carcinoma; ICU, intensive care unit; LT, liver transplantation; MELD, model for end-stage liver disease; PT-INR, prothrombin time international normalized ratio; SLV, standard liver volume; SMI, skeletal muscle mass index; SMlv, skeletal muscle mass index variation.

risk of graft loss, GR was analyzed as a clinically oriented categorical variable. Thus, low GR was defined as a GR lower than the 25 th percentile of the whole population.

\section{Results}

The study population comprised 106 patients and was characterized by a male-to-female ratio of $43: 63$ with a median age of 57 [50-62] years (Table 1). The median model for end-stage liver disease (MELD) score was 17 [13-23] and $9.4 \%$ of patients had already been admitted to intensive care unit (ICU) before LT. The median BMI was 22.9 (20.8-26.5) and the mean pre-LT SMI value for men and women was $47.2 \pm 9.1$ and $39.1 \pm 7.1$ respectively $(\mathrm{P}<0.001)$. A low muscle mass was diagnosed in 45 patients $(42.5 \%)$. The median graft 
Table 2 Analysis of potential correlations between GRR and recipient, donor or graft characteristics, intraoperative or postoperative details-continuous variables

\begin{tabular}{|c|c|c|}
\hline Variables & $\begin{array}{l}\text { Correlation } \\
\text { coefficient }\end{array}$ & $P$ \\
\hline Recipient age & -0.155 & 0.113 \\
\hline Recipient BMI & 0.097 & 0.322 \\
\hline \multicolumn{3}{|l|}{ Pre-LT SMI } \\
\hline Men & 0.352 & 0.020 \\
\hline Women & 0.138 & 0.290 \\
\hline \multicolumn{3}{|l|}{1 month post-LT SMI } \\
\hline Men & 0.172 & 0.271 \\
\hline Women & 0.076 & 0.560 \\
\hline \multicolumn{3}{|l|}{1 month post-LT SMlv\% } \\
\hline Men & -0.301 & 0.049 \\
\hline Women & -0.097 & 0.457 \\
\hline MELD & -0.114 & 0.245 \\
\hline Child-Pugh score & -0.076 & 0.439 \\
\hline Recipient SLV & 0.099 & 0.311 \\
\hline Donor age & -0.306 & 0.001 \\
\hline GV/SLV\% & -0.467 & $<0.001$ \\
\hline GRWR & -0.414 & $<0.001$ \\
\hline Operative time & 0.045 & 0.649 \\
\hline Blood loss & -0.096 & 0.327 \\
\hline Post-LT ICU length of stay & 0.042 & 0.672 \\
\hline 1 month post-LT bilirubin serum level & -0.014 & 0.885 \\
\hline 1 month post-LT PT-INR & -0.072 & 0.466 \\
\hline 1 month post-LT albumin plasma level & 0.072 & 0.471 \\
\hline 1 month post-LT cholinesterase serum level & 0.397 & 0.002 \\
\hline
\end{tabular}

BMI, body mass index; SMI, skeletal muscle mass index; SMIV\%, SMI variation; MELD, model for end-stage liver disease; GV/SLV\%, graft volume to recipient standard liver volume ratio; GRWR, graft to recipient weight ratio; ICU, intensive care unit; PT-INR, prothrombin time international normalized ratio.

volume-to-recipient standard liver volume percent (GV/ SLV\%) and graft-to-recipient weight ratio (GRWR) were $40 \%(36-47 \%)$ and $0.78(0.66-0.92)$ respectively. The donors were characterized by a median age of 34 [27-42] years, and in all cases the BMI was lower than 25.

At one month post-LT, male patients showed a mean
SMI of $43.8 \pm 8.9$ while women of $39.7 \pm 7.8$, with a SMIv\% of $-8.00 \%(-12.28 \%$ to $-3.67 \%)$ and $+2.55 \%(-5.30 \%$ to $+11.27 \%)$ respectively. The difference of SMIv\% was statistically significant $(\mathrm{P}<0.001)$. The prevalence of low muscle mass diagnosis decreased to $38.7 \%$, without a statistically significant difference compared to pre-LT prevalence $(\mathrm{P}=0.39)$. The median GRR at 1 month postLT was $90.90 \%$ (65.65-127.76\%). GV/SLV\%, GRWR and donor age showed a significant negative correlation with GRR (Tables 2-4). Conversely Pre-LT SMI showed a significant positive correlation in men (Spearman's rho $0.352, \mathrm{P}=0.02$ ) but not in women. Furthermore, in male recipients, GRR inversely correlated with the SMIv\% (Spearman's rho $-0.30, \mathrm{P}=0.05$ ) at a statistically significant level. Using the gender-specific cut-off values for low muscle mass diagnosis recommended by the Japanese Society of Hepatology guidelines (13), patients with preoperative low muscle mass showed a significantly lower GRR compared to patients with normal skeletal muscularity $(\mathrm{P}=0.04)$. Conversely, no association with 1 month post-LT low muscularity was noted. Multivariate linear regression analysis showed that GV/SLV\% and donor age independently correlated with GRR in the whole study population as well as in gender specific subgroups (R-squared $=0.44)$. Furthermore, in male recipients, pre-LT SMI and SMIv\% also maintained a statistically significant correlation with GRR (R-squared $=0.58$ ) (Tables 2-4). No correlation was registered between GRR and 1 month post-LT ratio of prothrombin time (PT-INR), bilirubin or albumin plasma level while cholinesterase serum level showed a significant positive correlation (Spearman's rho 0.397, $\mathrm{P}=0.002$ ).

Graft loss occurred in $8(7.5 \%)$ patients: 4 patients died because of graft failure while 4 were submitted to retransplantation. The underlying causes comprised HCV recurrence ( 3 pts), acute artery thrombosis ( $1 \mathrm{pt})$, acute massive portal thrombosis (1 pt), graft absessualization (1 pt), chronic graft rejection (1 pt) and tumor recurrence (1 pt). Low GRR was not shown to be a significant risk factor for graft loss (Table 5). Excluding retransplantation cases, the patient OS at 1,3 and 5 years was $90.1 \%, 86.6 \%$ and $78.6 \%$ respectively, and a low GRR was recorded in $23.6 \%$ of cases. In univariate analysis, low GRR, donor age, post-LT ICU length of stay, early post-LT blood stream infection, 1month post-LT bilirubin serum level and 1 month post-LT PT-INR were shown to be significant prognostic factors for OS (Table 6, Figure 2). In multivariate analysis, low GRR [HR 6.045; 95\% confidence interval (CI), 
Table 3 Analysis of potential correlations between GRR and recipient, donor or graft characteristics, intraoperative or postoperative detailscategorical variables

\begin{tabular}{|c|c|c|}
\hline Variables & 1 month post-LT GRR (\%) & $P$ \\
\hline Female & $89.78(67.45-113.8)$ & \\
\hline Recipient pre-LT diabetes & & 0.434 \\
\hline Absent & $93.19(67.92-134.22)$ & \\
\hline Pre-LT low muscle mass & & 0.041 \\
\hline Absent & $97.50(73.06-130.0)$ & \\
\hline Present & $83.52(45.20-110.9)$ & \\
\hline 1 month post-LT low muscle mass & & 0.514 \\
\hline Absent & $93.19(69.78-129.6)$ & \\
\hline Present & $82.93(50.03-113.6)$ & \\
\hline HCC diagnosis & & 0.748 \\
\hline Absent & $90.52(67.45-129.6)$ & \\
\hline Present & $92.65(64.10-117.7)$ & \\
\hline Pre-transplant ICU admission & & 0.729 \\
\hline Absent & $91.60(64.10-128.6)$ & \\
\hline Present & $85.49(69.78-105.2)$ & \\
\hline Present & $84.81(73.06-102.5)$ & \\
\hline Early biliary complications & & 0.625 \\
\hline Absent & $89.22(65.34-127.7)$ & \\
\hline Present & $95.75(79.00-121.1)$ & \\
\hline Early vascular complications & & 0.193 \\
\hline Absent & $91.07(65.55-129.6)$ & \\
\hline Present & $75.90(62.67-97.50)$ & \\
\hline Early blood stream infection & & 0.852 \\
\hline Absent & $90.52(65.34-124.8)$ & \\
\hline Present & $91.98(68.03-167.4)$ & \\
\hline Early rejection & & 0.758 \\
\hline Absent & $90.90(66.50-126.3)$ & \\
\hline Present & 95.38 (55.39-137.9) & \\
\hline
\end{tabular}

LT, liver transplantation; HCC, hepatocellular carcinoma; HCV, hepatitis C virus; ICU, intensive care unit. 
Table 4 Multivariate linear regression analysis of predictors of GRR

\begin{tabular}{|c|c|c|c|}
\hline Factors & Regression coefficient & $95 \% \mathrm{Cl}$ & $P$ value \\
\hline Pre-LT low muscle mass & -8.563 & -26.301 to +9.175 & 0.341 \\
\hline Donor age & -1.200 & -1.899 to -0.501 & 0.001 \\
\hline GV/SLV\% & -443.017 & -667.981 to -218.054 & $<0.001$ \\
\hline \multicolumn{4}{|l|}{ Men } \\
\hline Pre-LT SMI & 1.734 & +0.451 to +3.018 & 0.009 \\
\hline 1 month post-LT SMIv\% & -1.190 & -2.227 to -0.153 & 0.026 \\
\hline Donor age & -1.309 & -2.240 to -0.379 & 0.007 \\
\hline \multicolumn{4}{|l|}{ Women } \\
\hline Donor age & -1.220 & -2.178 to -0.263 & 0.013 \\
\hline GV/SLV\% & -428.970 & -695.458 to -162.483 & 0.002 \\
\hline GRWR & 49.788 & -57.892 to +157.469 & 0.359 \\
\hline
\end{tabular}

LT, liver transplantation; GV/SLV\%, graft volume to recipient standard liver volume ratio; GRWR, graft to recipient weight ratio; SMI, skeletal muscle mass index; SMIv\%, SMI variation.

2.378-15.367, $\mathrm{P}<0.001$ ], 1 month post-LT PT-INR (HR $5.818,95 \%$ CI $2.048-16.528, \mathrm{P}=0.001)$ and ICU length of stay (HR 1.105; 95\% CI, 1.069-1.143, $\mathrm{P}<0.001$ ) maintained statistical significance.

\section{Discussion}

GR is a highly complex biological process which is activated and regulated by multiple inflammatory, growth and metabolic pathways $(6,10,11,16)$. In partial grafts, the increased shear stress associated with portal hyperperfusion activates an inflammatory reaction with release of vascular endothelial growth factor, interleukin 6, and nitric oxide by Kupffer cells and sinusoidal endothelial cells $(8,9)$. These mediators have been demonstrated to activate replication processes in the hepatocytes $(8,9)$. Clinically, several parameters of splanchnic circulation and portal flow $(8,9,17)$ as well as parameters of graft-to-recipient relative mass (GV/SLV and GRWR) have been demonstrated to strongly correlate with the GR. In the present study, GV/SLV and GRWR were the variables with the highest correlation coefficient. Regarding the graft quality, advanced donor age and graft steatosis have been also identified as significant risk factors for GR $(10,11,18-20)$ The aging is associated with a progressive loss of hepatocytes regenerative capacity, characterized by a decrease of cell cycle and increase of autophagy and apoptosis $(10,11,21,22)$, while steatotic grafts show a greater susceptibility to ischemia-reperfusion injury $(7,10,11)$. In the present study, the recipient age did have a significant and negative impact on GR. Such result may have also been determined by a $16 \%$ prevalence of donors older than 50 years, which is the most frequently reported cut-off for a higher risk of poor graft outcome (10). Nonetheless, the analyses of the outcomes in donorrecipient pairs has failed to demonstrate any correlation between the two parts of the same liver in terms of regenerative behavior, indicating that the host plays a significant role in driving the process (16). As a matter of fact, the clinical conditions of the recipients have emerged as a critical determinant of GR, although the available data on the underlying pathogenesis and mechanism are very limited. The ischemic stress and metabolic demands in recipients appear to provide a growth stimulus to activate priming cytokines for initiation of liver graft regeneration $(6,11,16)$. However, when the severity of the recipient illness is too advanced, susceptible grafts 
Table 5 Univariate analysis of prognostic factors for graft loss risk

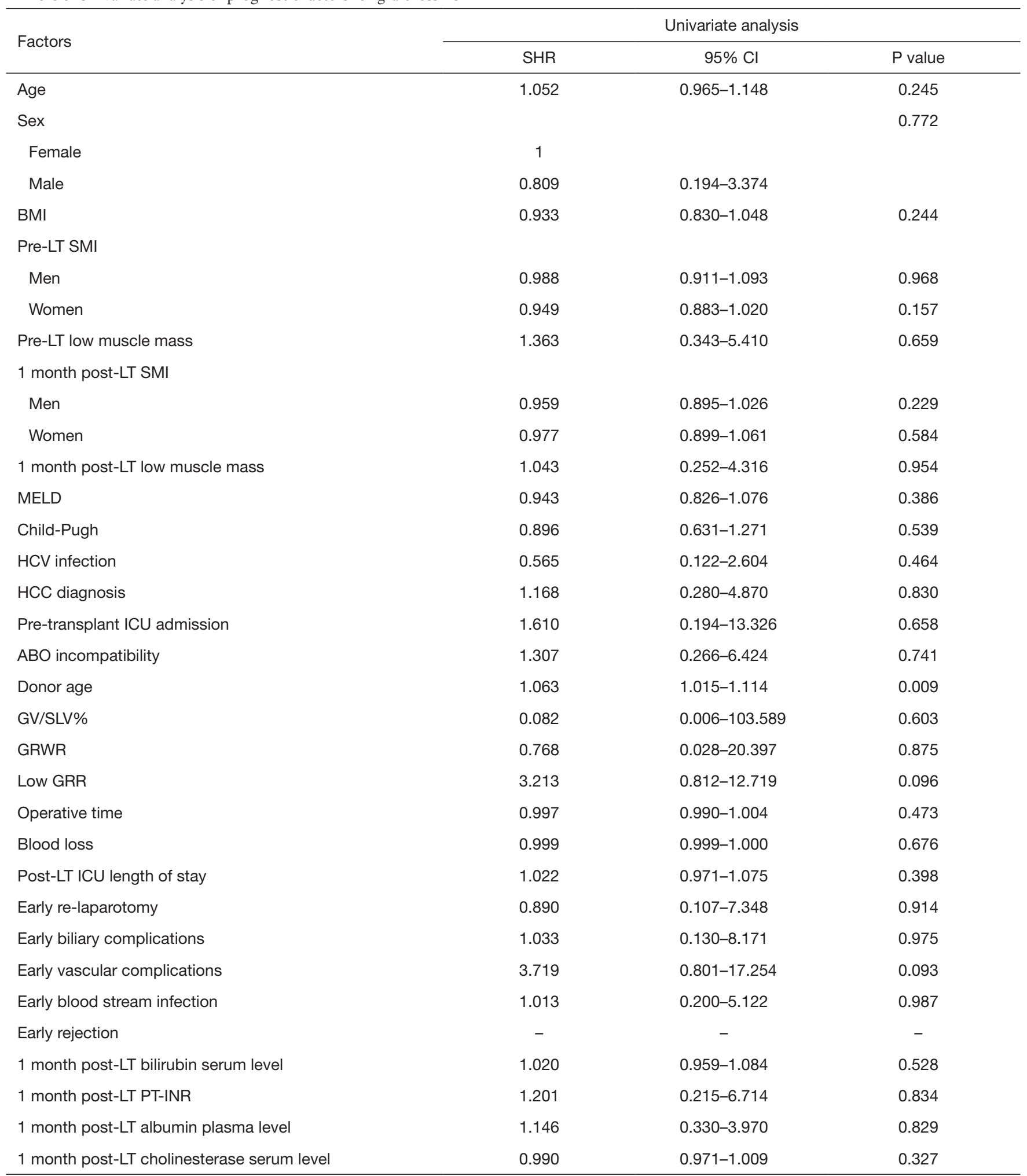

BMI, body mass index; GRR, graft regeneration rate; GRWR, graft to recipient weight ratio; GV/SLV, graft volume to recipient standard liver volume ratio; HCV, hepatitis C virus; HCC, hepatocellular carcinoma; ICU, intensive care unit; LT, liver transplantation; MELD, model for end-stage liver disease; PT-INR, prothrombin time international normalized ratio; SHR, subhazard ratio; SMI, skeletal muscle mass index. 
Table 6 Univariate Cox analysis of prognostic factors for patient OS

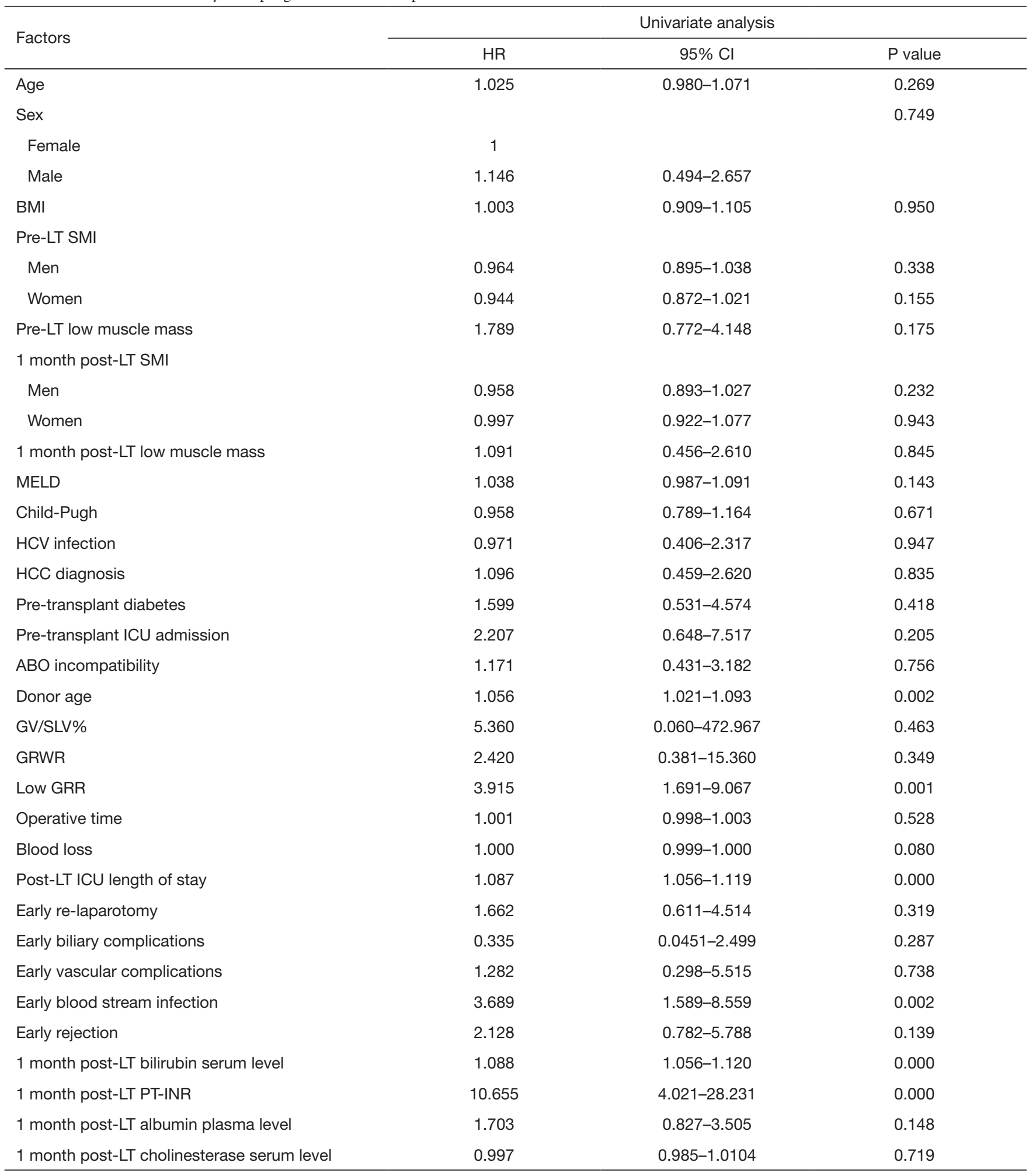

BMI, body mass index; GRR, graft regeneration rate; GRWR, graft to recipient weight ratio; GV/SLV\%, graft volume to recipient standard liver volume ratio; HCV, hepatitis C virus; HCC, hepatocellular carcinoma; HR, hazard ratio; ICU, intensive care unit; LT, liver transplantation; MELD, model for end-stage liver disease; PT-INR, prothrombin time international normalized ratio; SMI, skeletal muscle mass index. 


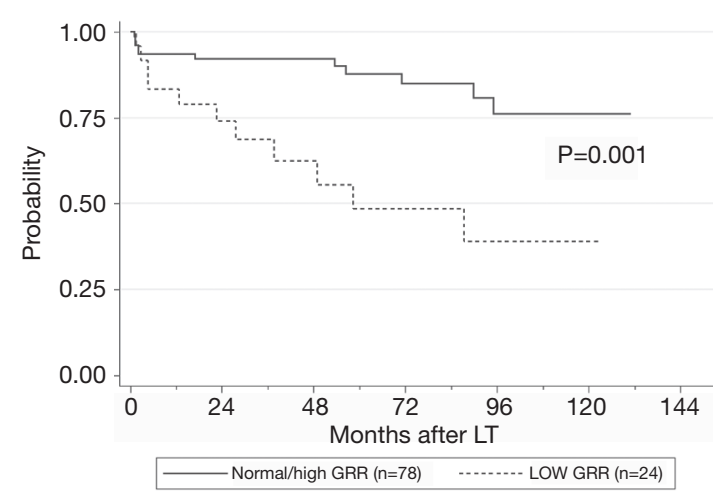

Figure 2 Overall survival curves after LDLT comparing recipients with low GRR and normal/high GRR. LDLT, living-donor liver transplantation; GRR, graft regeneration rate.

may fail to sustain the energy and metabolic burden or to recover from the ischemic-reperfusion injury and portal hyperperfusion injury, thus resulting in an impaired regeneration and functional decompensation $(6,11,16,23)$. Therefore, the graft must balance the available resources between the recipient's metabolic homeostasis and its regeneration. In the present study, low muscle mass as defined by the Japan Society of Hepatology (13) was associated with a significantly lower GRR in both sexes. However just in men it was possible to record a significant and positive correlation between GRR and pre-LT SMI. It has been verified that malnutrition in male patients with ESLD is mainly characterized by muscle mass loss, whereas that in women is characterized by fat loss (1). Furthermore, ESLD male patients frequently suffer of hypogonadism with low testosterone levels which further worsen muscle mass degeneration and loss (24). Identifying male LT candidates as having a higher risk of poor energy and proteins reserve may explain why pre-LT SMI did directly correlate with GR specifically in men. Furthermore, as we previously reported (12), male recipients tend to have a worsening of their skeletal muscle mass in the early postLT period, compared to a substantial stable trend in women. Demonstrating in the present study that in men the greater was the GRR, the lower was the SMIv\%, may indicate that the graft and the skeletal muscles somehow competed for the same metabolic substrates. This result was surprising as it might be expected that the graft regeneration could sustain the muscle mass recovery rather than further compromise it. Conversely, it appeared that the probable limited availability of metabolic substrates made necessary to preferentially shunt the metabolism toward hepatocytes replication pathways, to the detriment of the skeletal muscle fibers. Further studies are needed anyway to clarify this potential adaptive mechanism.

EEF protocols after LDLT have been already demonstrated to control the negative impact of pre-LT low muscularity and sarcopenia on post-LT recipients survival but currently fail to significantly improve the skeletal muscle mass (12). These findings were confirmed even by the present investigation which also showed that EEF was apparently not sufficient to counteract the preLT metabolic dysfunction and supplement the recipients' metabolic reserves.

A direct correlation between graft volume and laboratory markers of liver function has been verified mainly in the early post-LT period in LDLT (6) when small-forsize grafts or grafts with a low regenerative reactivity are associated with a higher risk of early dysfunction $(6,11)$. However, in more advanced postoperative phases, it has been reported that the early allograft dysfunction acts as a trigger for a greater GR to compensate the metabolic deficit of the early post-LT period (6). Moreover, many therapeutic interventions, such as albumin supplementation, vitamin $\mathrm{K}$ administration or biliary drainage, as well as post-LT complications such as biliary complications may alter the direct correlation between graft regeneration and function. As a matter of fact, in the present study GRR did show a significant correlation, among all the examined laboratory liver function markers at 1 month post-LT, only with cholinesterase serum levels, which are not clinically modifiable. Nonetheless a low GRR as well as the laboratory markers of graft function (bilirubin serum level, PT-INR) were both found to be significant determinants of recipient survival. These results are in line with previous reports (25). No correlation was noted between GRR and graft survival, probably due to the underlying causes of graft loss which appeared not to be directly correlated with a poor GR.

Several limitations must be mentioned for this study: no data on insulin resistance during the early postoperative period; unavailability of specific values of $\mathrm{Kcal} / \mathrm{kg}$ and protein $/ \mathrm{kg}$ administered to the recipients during the post-LT course, despite none of the patients required a withdrawal of the EEF protocol and the calories intake was monitored and maintained over 1,200 kcal/day during all the hospital stay; a low-moderate statistical strength of the GRR correlation indices, although in line with previous 
reports $(8,11)$; the retrospective modality of the data analysis and limited number of patients in the study population.

\section{Conclusions}

GR is crucial in LDLT to normalize the portal hypertension and sustain the metabolic demand of the recipients, and a low GRR at 1 month post-LT is associated with a poor recipient overall survival. Additionally to the hemodynamic factors of portal circulation and the quality of the graft, even the metabolic status of the recipients seems to have a significant role in the GR process. A pre-LT low skeletal muscle mass is associated with impaired GR and this negative impact is more evident in men whose metabolic dysfunction tends to be more severe, despite the use of EEF protocols. New strategies or formulas of nutritional interventions may probably be warranted to treat sarcopenia and the related underlying metabolic dysfunction before and early after LDLT.

\section{Acknowledgments}

Funding: None.

\section{Footnote}

Conflicts of Interest: All authors have completed the ICMJE uniform disclosure form (available at https://hbsn. amegroups.com/article/view/10.21037/hbsn.2019.11.08/coif). The authors have no conflicts of interest to declare.

Ethical Statement: The authors are accountable for all aspects of the work in ensuring that questions related to the accuracy or integrity of any part of the work are appropriately investigated and resolved. The study was approved by the local Institutional Review Board.

Open Access Statement: This is an Open Access article distributed in accordance with the Creative Commons Attribution-NonCommercial-NoDerivs 4.0 International License (CC BY-NC-ND 4.0), which permits the noncommercial replication and distribution of the article with the strict proviso that no changes or edits are made and the original work is properly cited (including links to both the formal publication through the relevant DOI and the license). See: https://creativecommons.org/licenses/by-nc$\mathrm{nd} / 4.0 /$.

\section{References}

1. Kalafateli M, Mantzoukis K, Choi Yau Y, et al. Malnutrition and sarcopenia predict post-liver transplantation outcomes independently of the Model for End-stage Liver Disease score. J Cachexia Sarcopenia Muscle 2017;8:113-21.

2. Sinclair M, Gow PJ, Grossmann M, et al. Review article: sarcopenia in cirrhosis--aetiology, implications and potential therapeutic interventions. Aliment Pharmacol Ther 2016;43:765-77.

3. Mazurak VC, Tandon P, Montano-Loza AJ. Nutrition and the transplant candidate. Liver Transpl 2017;23:1451-64.

4. Hammad A, Kaido T, Aliyev V, et al. Nutritional Therapy in Liver Transplantation. Nutrients 2017;9.

5. Dasarathy S. Post transplant sarcopenia: an under recognized early consequence of liver transplantation.Dig Dis Sci 2013;58:3103-11.

6. Chae MS, Kim Y, Lee N, et al. Graft Regeneration and Functional Recovery in Patients with Early Allograft Dysfunction After Living-Donor Liver Transplantation. Ann Transplant 2018;23:481-90.

7. Chae MS, Lee N, Choi HJ, et al. Comparison of Liver Graft Regeneration Between ABO-Compatible and ABOIncompatible Living Donor Liver Transplantation: A Propensity Score Matching Analysis. Ann Transplant 2018;23:507-19.

8. Byun SH, Yang HS, Kim JH. Liver graft hyperperfusion in the early postoperative period promotes hepatic regeneration 2 weeks after living donor liver transplantation: A prospective observational cohort study. Medicine (Baltimore) 2016;95:e5404.

9. Jiang SM, Zhou GW, Zhang R, et al. Role of splanchnic hemodynamics in liver regeneration after living donor liver transplantation. Liver Transpl 2009;15:1043-9.

10. Taniguchi M, Shimamura T, Todo S, Furukawa H. Smallfor-size syndrome in living-donor liver transplantation using a left lobe graft. Surg Today 2015;45:663-71.

11. Tanemura A, Mizuno S, Wada H, et al. Donor age affects liver regeneration during early period in the graft liver and late period in the remnant liver after living donor liver transplantation. World J Surg 2012;36:1102-11.

12. Pravisani R, Soyama A, Isola M, et al. Chronological changes in skeletal muscle mass following living-donor liver transplantation: An analysis of the predictive factors for long-term post-transplant low muscularity. Clin Transplant 2019;33:e13495.

13. Nishikawa H, Shiraki M, Hiramatsu A, et al. Japan Society of Hepatology guidelines for sarcopenia in liver disease 
(1st edition): Recommendation from the working group for creation of sarcopenia assessment criteria. Hepatol Res 2016;46:951-63.

14. Akamatsu N, Sugawara Y, Nagata R, et al. Adult right living-donor liver transplantation with special reference to reconstruction of the middle hepatic vein. Am J Transplant 2014;14:2777-87.

15. Hara T, Soyama A, Hidaka M, et al. Analysis of early relaparotomy following living donor liver transplantation. Liver Transpl 2016;22:1519-25.

16. Olthoff KM, Emond JC, Shearon TH, et al. Liver regeneration after living donor transplantation: adult-toadult living donor liver transplantation cohort study. Liver Transpl 2015;21:79-88.

17. Eguchi S, Yanaga K, Sugiyama N, et al. Relationship between portal venous flow and liver regeneration in patients after living donor right-lobe liver transplantation. Liver Transpl 2003;9:547-51.

18. Cheng YF, Huang TL, Chen TY, et al. Liver graft regeneration in right lobe adult living donor liver transplantation. Am J Transplant 2009;9:1382-8.

19. Ikegami T, Nishizaki T, Yanaga K, et al. The impact

Cite this article as: Pravisani R, Soyama A, Ono S, Baccarani U, Isola M, Takatsuki M, Hidaka M, Adachi T, Hara T, Hamada T, Pecquenard F, Risaliti A, Eguchi S. Is there any correlation between liver graft regeneration and recipient's pretransplant skeletal muscle mass? - a study in extended left lobe graft living-donor liver transplantation. HepatoBiliary Surg Nutr 2020;9(2):183-194. doi: 10.21037/hbsn.2019.11.08 of donor age on living donor liver transplantation. Transplantation 2000 Dec 27;70:1703-7.

20. Lué A, Solanas E, Baptista P, et al. How important is donor age in liver transplantation? World J Gastroenterol 2016;22:4966-76.

21. Schmucker DL, Sanchez H. Liver regeneration and aging: a current perspective. Curr Gerontol Geriatr Res 2011;2011:526379.

22. Enkhbold C, Morine Y, Utsunomiya T, et al. Dysfunction of liver regeneration in aged liver after partial hepatectomy. J Gastroenterol Hepatol 2015;30:1217-24.

23. Wolf JH, Holmes MV, Fouraschen S et al. Serum lipid expression correlates with function and regeneration following living donor liver transplantation. Liver Transpl 2016;22:103-10.

24. Sinclair M, Grossmann M, Gow PJ, et al. Testosterone in men with advanced liver disease: abnormalities and implications. J Gastroenterol Hepatol 2015;30:244-51.

25. Muraoka N, Uematsu H, Kimura H, et al. Rate of liver volume increase: can it predict recipient prognosis after left lobe transplantation from living related donors? Clin Imaging 2008;32:6-10. 